\title{
2-micron triple-pulse integrated path differential absorption lidar development for simultaneous airborne column measurements of carbon dioxide and water vapor in the atmosphere
}

\author{
Upendra N. Singh, Mulugeta Petros, Tamer F. Refaat and Jirong Yu \\ NASA Langley Research Center, 11 Langley Blvd., Hampton, VA 23681, USA
}

\begin{abstract}
For more than 15 years, NASA Langley Research Center (LaRC) has contributed in developing several 2-micron carbon dioxide active remote sensors using the DIAL technique. Currently, an airborne 2-micron triple-pulse integrated path differential absorption (IPDA) lidar is under development at NASA LaRC. This paper focuses on the advancement of the 2-micron triple-pulse IPDA lidar development. Updates on the state-of-the-art triple-pulse laser transmitter will be presented including the status of wavelength control, packaging and lidar integration. In addition, receiver development updates will also be presented, including telescope integration, detection systems and data acquisition electronics. Future plan for IPDA lidar system for ground integration, testing and flight validation will be presented.
\end{abstract}

Keywords: Active remote sensing, carbon dioxide, water vapor, DIAL, IPDA lidar, triple-pulse laser

\section{INTRODUCTION}

The $2.0 \mu \mathrm{m}$ wavelength region is suitable for atmospheric carbon dioxide $\left(\mathrm{CO}_{2}\right)$ measurements due to the existence of distinct absorption feathers for the gas at this particular wavelength. For more than 20 years, researchers at NASA Langley Research Center (LaRC) have developed several high-energy and high repetition rate 2- $\mu \mathrm{m}$ pulsed lasers. Operating as radiation transmitters, these lasers are the key component in several lidar instrumentation for atmospheric wind and carbon dioxide $\left(\mathrm{CO}_{2}\right)$ measurements [1]. Applying the differential absorption lidar (DIAL) technique, LaRC has demonstrated several $\mathrm{CO}_{2}$ active remote sensors using the $2-\mu \mathrm{m}$ laser for the last 15 years. Both range-resolved (RR) DIAL and integrated-path differential absorption (IPDA) lidar have been attempted [1]. $\mathrm{CO}_{2}$ RR-DIAL measurements using single-pulse $2-\mu \mathrm{m}$ laser have been demonstrated using both coherent and direct-detection techniques [2-4]. In single-pulse DIAL, the wavelength of the transmitted laser pulses alternate between on- and off-line positions at a relatively slow rate $(5-10 \mathrm{~Hz})$. Using coherent detection, vertical range-resolved $\mathrm{CO}_{2}$ measurements were made with $2.4 \%$ precision using $500 \mathrm{~m}$ range bins and 6.7 minute integration time. Measurement of horizontal $\mathrm{CO}_{2}$ column indicated a precision of less than $0.7 \%$ using a 30 minute integration time [2]. Byproducts of this system include wind and aerosols measurements with the same lidar instrument. Detection technology at 2- $\mu \mathrm{m}$ limits the $\mathrm{CO}_{2} \mathrm{RR}-\mathrm{DIAL}$ profiling capability. Therefore, hetero-junction phototransistors have been developed and demonstrated for the first time in a 2- $\mu \mathrm{m}$ lidar [3-4]. Using phototransistor-based direct detection, another 2- $\mu \mathrm{m} \mathrm{CO}_{2}$ RR-DIAL system was developed at NASA LaRC using the same transmitter [3]. Field experiments with this instrument indicated average boundary layer $\mathrm{CO}_{2}$ mixing ratios of $390 \mathrm{ppm}$ with $3.0 \%$ precision. Experiments also demonstrated the $2-\mu \mathrm{m}$ DIAL capability of measuring atmospheric $\mathrm{CO}_{2}$ integrated column content [4]. Although $\mathrm{CO}_{2}$ DIAL systems demonstrations were provided as a proof of concept for systems validity from ground or airborne platforms, a complete active remote sensing mission that contributes to the science community has not been established yet.

Double-pulsed 2- $\mu \mathrm{m}$ lasers have been developed with energy as high as $600 \mathrm{~mJ}$ and up to $10 \mathrm{~Hz}$ shot repetition rate [5]. This laser produces two successive pulses, per laser shot, which can be tuned and locked independently at two different wavelengths. These capabilities are well suited for IPDA lidar transmitters. IPDA lidar relies on strong hard target return signals that provide higher signal-to-noise ratio (SNR) measurement compared to conventional RR-DIAL, which depends on weaker atmospheric scattering. IPDA lidar systems are suitable for airborne measurements while operating in nadir mode. Recently, an airborne $\mathrm{CO}_{2}$ double-pulsed 2- $\mu$ m IPDA lidar was developed at NASA LaRC [6-9]. The instrument was validated on ground for atmospheric $\mathrm{CO}_{2}$ measurement using calibrated hard targets before integration onboard the NASA B-200 aircraft [7-8]. Airborne validation experiments for atmospheric $\mathrm{CO}_{2}$ measurements using this IPDA lidar included different conditions with both land and ocean targets [9]. Currently, work efforts are focus on 
enhancing the capability of this instrument through triple-pulse operation [10-11]. Accommodating a third transmitted pulse would target water vapor $\left(\mathrm{H}_{2} \mathrm{O}\right)$, the more abundant and most interfering molecule on $\mathrm{CO}_{2}$ measurement [11]. This paper presents the measurement concept, current status and potential validation plans for the airborne 2- $\mu \mathrm{m}$ triple-pulse IPDA lidar instrument development at NASA LaRC for atmospheric $\mathrm{CO}_{2}$ and $\mathrm{H}_{2} \mathrm{O}$ simultaneous and independent measurements.

\section{TRIPLE-PULSE IPDA REMOTE SENSING TECHNIQUE}

The $2 \mu \mathrm{m}$ wavelength region offers many suitable absorption features for $\mathrm{CO}_{2}$ as well as $\mathrm{H}_{2} \mathrm{O}$ [11]. For $\mathrm{CO}_{2}$ sensing, the R30 line, located at $2050.9670 \mathrm{~nm}$, provides an attractive absorption cross section with unique characteristics. These include low temperature sensitivity and low interference from higher abundant $\mathrm{H}_{2} \mathrm{O}$ molecules. This is indicated in figure 1, which compares the composite absorption spectra for $\mathrm{CO}_{2}$ and $\mathrm{H}_{2} \mathrm{O}$. The spectra were derived using the HITRAN database for line parameters, assuming Voigt line profile at two altitudes [19]. Altitude selection is based on IPDA instrument operation limits. Zero for ground-based evaluation and $8 \mathrm{~km}$ for maximum airborne altitude validation onboard the NASA B-200 aircraft. Highest intensity lines were included in these calculations. This results in 708 and 550 lines, spanning from 2000.0002 to $2099.3019 \mathrm{~nm}$ and from 2050.0009 to $2051.9946 \mathrm{~nm}$ for water vapor and carbon dioxide, respectively. The $\mathrm{H}_{2} \mathrm{O}$ absorption peak located at $2050.5322 \mathrm{~nm}$ coincides close to $\mathrm{CO}_{2}$ absorption minima, between R30 and R32 lines and away from the R32 peak. The IPDA laser transmitter generates three successive pulses locked to three different wavelengths, as shown schematically in figure 2. A single pump pulse produces three successive 2- $\mu \mathrm{m}$ laser pulses, $150 \mu \mathrm{sec}$ apart. Using an enhanced wavelength control scheme, each of these pulses can be tuned and locked at different wavelength, as marked in figure 1 . The principle of wavelength selection for this IPDA instrument is demonstrated in the same figure. The $\mathrm{CO}_{2}$ on and off-line wavelengths are selected around the $\mathrm{R} 30$ line, so that both would have similar $\mathrm{H}_{2} \mathrm{O}$ absorption (see lower horizontal line). This minimizes the $\mathrm{H}_{2} \mathrm{O}$ interference on the $\mathrm{CO}_{2}$ measurements. Similarly, the $\mathrm{H}_{2} \mathrm{O}$ on- and off-line are selected around the absorption peak such that $\mathrm{CO}_{2}$ interference is minimized on the $\mathrm{H}_{2} \mathrm{O}$ measurement (see upper horizontal line). However, both $\mathrm{CO}_{2}$ and $\mathrm{H}_{2} \mathrm{O}$ measurements share the same wavelength (i.e., the same pulse) for on- and off-line, respectively. This allows achieving simultaneous measurement of both molecules with triple pulses rather than quadruple pulses almost independently while avoiding interference from each other [11].

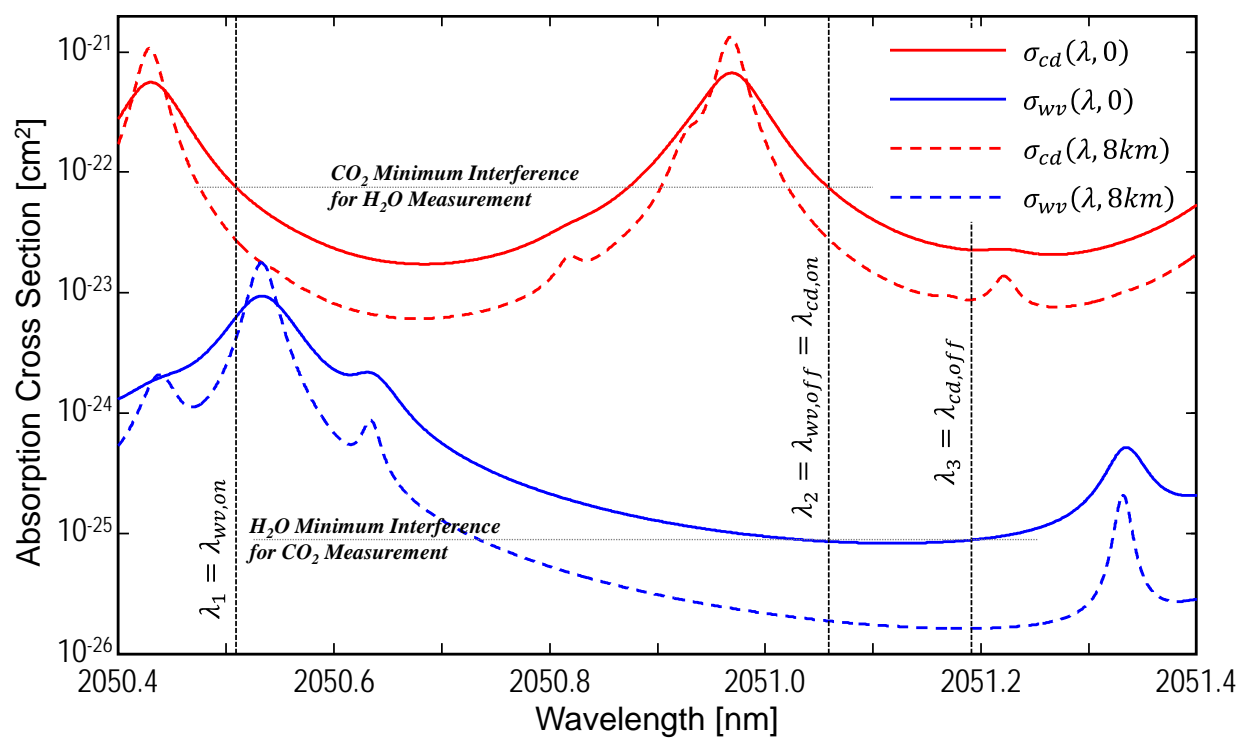

Figure 1. Comparison of the $\mathrm{H}_{2} \mathrm{O}$ and $\mathrm{CO}_{2}$ absorption cross-section spectra, $\sigma_{\mathrm{wv}}$ and $\sigma_{\mathrm{cd}}$, respectively, at ground $(0 \mathrm{~km})$ and mid-altitude aircraft $(8 \mathrm{~km})$. Temperature and pressure profiles used in the calculation were obtained from the US Standard atmospheric model. Vertical lines mark the instrument operating wavelengths for $\mathrm{CO}_{2}$ and $\mathrm{H}_{2} \mathrm{O}$ independent measurements. Note that $\lambda_{1}$ is the $\mathrm{H}_{2} \mathrm{O}$ on-line, $\lambda_{2}$ serves as the $\mathrm{H}_{2} \mathrm{O}$ off-line, and the $\mathrm{CO}_{2}$ on-line and $\lambda_{3}$ is the $\mathrm{CO}_{2}$ off-line. The horizontal lines mark the absorption cancellation for minimizing molecular interference [11]. 


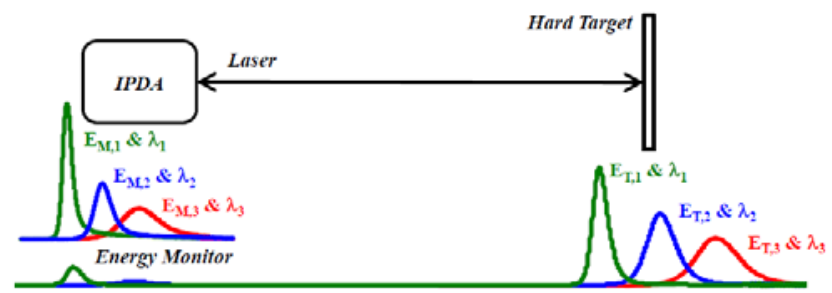

Figure 2. Principle of operation of the 2- $\mu \mathrm{m}$ triple-pulse IPDA lidar for atmospheric $\mathrm{CO} 2$ and $\mathrm{H} 2 \mathrm{O}$ measurement. The wavelength selection in based on the criteria presented in figure 1 .
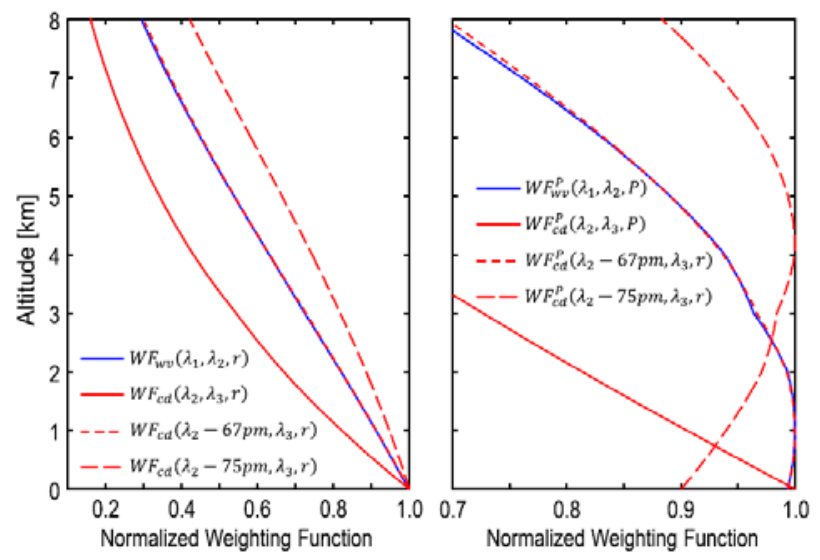

Figure 3. $\mathrm{CO}_{2}$ and $\mathrm{H}_{2} \mathrm{O}$ range-based (left) and pressure-based (right) weighting functions versus altitude at selected spectral positions for an airborne nadir pointing IPDA measurement. $\mathrm{H}_{2} \mathrm{O}$ and $\mathrm{CO}_{2}$ measurements are weighted to ABL and near the surface, respectively. Tuning the $\mathrm{CO}_{2}$ on-line wavelength 67 and $75 \mathrm{pm}$ away from the selected location would optimize the IPDA measurement to within the boundary layer or free troposphere [11].

To examine the measurement principle of the triple-pulse IPDA, radiative transfer calculations were conducted using the US Standard atmospheric model for meteorological and gas mixing ratio profiles. The $\mathrm{CO}_{2}$ mixing ratio model is modified based on the current ground nominal value of $397 \mathrm{ppm}$. It should be noted that the IPDA lidar technique works best for relatively well-mixed atmospheric gases, such as carbon dioxide. In this case, the weighted-average column dryair mixing ratio $\left(\mathrm{X}_{\mathrm{cd}}\right)$ closely represents the dry-air mixing ratio profile, $\mathrm{x}_{\mathrm{cd}}$, which is almost constant versus altitude. On the contrary, water vapor is a dynamic atmospheric gas, which is not well mixed. Therefore, $X_{\mathrm{wv}}$ is the fixed amount of water vapor that has the same absorption strength equivalent to the actual water vapor distributed dry-air mixing ratio profile, $\mathrm{x}_{\mathrm{wv}}$, in the measured column weighted by a defined weighting function, $\mathrm{WF}_{\mathrm{wv}}$, as shown in figure 3 .

\section{IPDA INSTRUMENT DEVELOPMENT}

Through continual support from the NASA Earth Science Technology Office (ESTO), current efforts at NASA LaRC are focused on realizing and developing the $2-\mu \mathrm{m}$ triple-pulse IPDA lidar instrument for independent and simultaneous monitoring of both atmospheric $\mathrm{CO}_{2}$ and $\mathrm{H}_{2} \mathrm{O}$. The triple-pulse IPDA instrument design, development and integration is based on the knowledge gathered through the successful demonstration of the double-pulse IPDA lidar for atmospheric $\mathrm{CO}_{2}$ measurements [6-11]. The triple-pulse IPDA lidar instrument consists of a 2- $\mu \mathrm{m}$ pulsed laser transmitter and a receiver. Several enhancements and updates of the triple-pulse instrument over the double-pulse instrument included in both the transmitter and the receiver systems. These include the ability of generating three successive pulses, instead of two, with high-energy (up to $70 \mathrm{~mJ}$ ) and high-repetition rate $(50 \mathrm{~Hz})$ using a single pump pulse. In addition, wavelength control and locking is extend further, up to $35 \mathrm{GHz}$ away from the $\mathrm{R} 30 \mathrm{CO}_{2}$ line, to accommodate $\mathrm{H}_{2} \mathrm{O}$ sensing. In the receiver, detection systems and data acquisition hardware are updated for better performance in terms of detection sensitivity and error reduction. Implementing real-time data analysis and processing would expedite science data production to further extend instrument testing and validation. These enhancements are detailed in the following subsections. 


\subsection{IPDA transmitter}

The triple-pulse IPDA laser transmitters is based on the Ho:Tm:YLF high-energy 2- $\mu \mathrm{m}$ pulsed laser technology. This laser transmitter is end pumped by AlGaAs diode arrays at $792 \mathrm{~nm}$ and designed to operate in a unique triple pulse format to mitigate the effect of the surface reflection difference between the different operating wavelength pulses on the precision of the IPDA measurement. Q-switch triple-trigger, relative to the pump pulse, produces three successive $2-\mu \mathrm{m}$ laser pulses with relatively controlled energies and pulse-widths. The objective is to set the first pulse $\left(\lambda_{1}\right)$ to higher energy of $50 \mathrm{~mJ}$ to accommodate $\mathrm{H}_{2} \mathrm{O}$ high variability absorption loss through on-line tuning. The second pulse $\left(\lambda_{2}\right)$ is set to lower energy of $15 \mathrm{~mJ}$ to accommodate $\mathrm{CO}_{2}$ absorption loss. In addition the same pulse would act as the $\mathrm{H}_{2} \mathrm{O}$ offline wavelength. The third pulse $\left(\lambda_{3}\right)$ with the lowest energy setting of $5 \mathrm{~mJ}$ would serve as the $\mathrm{CO}_{2}$ off-line. The three pulses are separated by approximately $200 \mu \mathrm{sec}$, in which minor atmospheric variability can be neglected for IPDA applications. Thermal analysis indicated the challenging task of proper heat dissipation rate out of the laser crystal. This is a critical issue that can result in crystal damage. A prototype oscillator with triple pulsing capability was demonstrated, as shown in figure 4, and final thermal analysis and alignment optimization is currently going on. In addition, a laser timing control (LTC) unite was designed, fabricated and tested. This unit controls several aspects of the laser operation with a computer interface that allows the operator to change settings through a graphical user interface (GUI). Three additional LTC units are being assembled to be used for prototype laser and spare. This unit is three times lighter than what was developed in double-pulsed IPDA.

The exact wavelengths of the pulsed laser transmitter are controlled by a wavelength control unit within $1 \mathrm{MHz}$. The unique wavelength control of the triple pulses uses a single seed semiconductor laser (JPL) and provides any frequency setting within $35 \mathrm{GHz}$ from the $\mathrm{CO}_{2} \mathrm{R} 30$ line center. The wavelength control unit includes several components which were acquired and characterized. These components include electro-optics modulator, circulator, fiber-based band-pass optical filters, and fiber amplifier. The wavelength control unit is designed to inject the three different wavelengths at the $200 \mu \mathrm{sec}$ pulse intervals for seeding. In this scheme, all three wavelengths are offset-locked in reference to the $\mathrm{CO}_{2} \mathrm{R} 30$ line center for a longtime frequency jitter of less than $300 \mathrm{kHz}$. Wavelength switching is accomplished in nanoseconds. The seed laser output is split into $10 \%$ and $90 \%$ beams. The $10 \%$ beam is modulated with a $200 \mathrm{MHz}$ electro-optics modulator and directed to a $\mathrm{CO}_{2}$ absorption cell for reference wavelength generation. Once the centerline is locked, the $90 \%$ beam passes through an electro-optic phase modulator to produce the required side lines within 2 to $35 \mathrm{GHz}$ from the center reference wavelength. The $\mathrm{CO}_{2}$ online and $\mathrm{H}_{2} \mathrm{O}$ off-line share a $6.5 \mathrm{GHz}$ offset frequency $\left(\lambda_{2}\right.$ in Fig. 1). The $\mathrm{CO}_{2}$ off-line $\left(\lambda_{3}\right)$ and $\mathrm{H}_{2} \mathrm{O}$ on-line $\left(\lambda_{1}\right)$ are offset 16 and $32 \mathrm{GHz}$, respectively. The wavelength of interest is discriminated using a fiber filter to suppress the centerline along with the undesired side mode and passes through a fiber amplifier to seed the oscillator. The integration of a prototype wavelength controller demonstrated technological challenges handling the high bandwidth of the signals which was managed by proper RF designs and implementations.
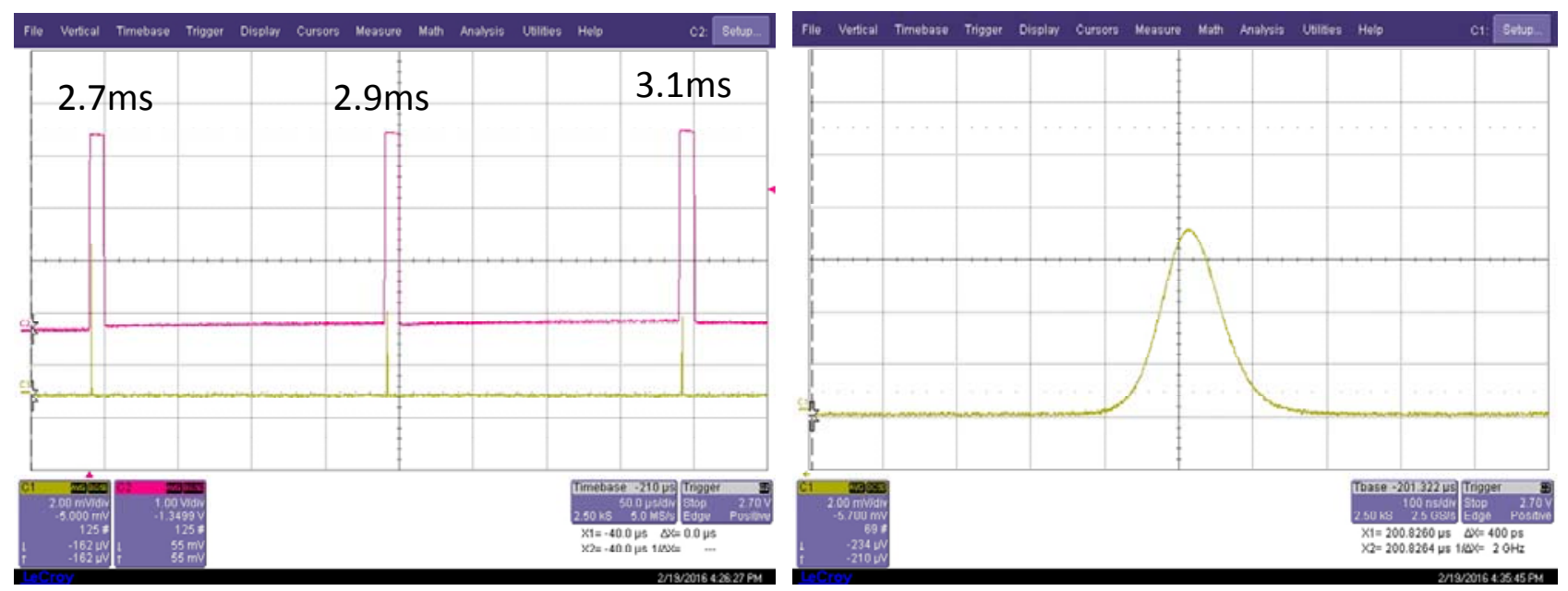

Figure 4. Oscilloscope records for the successful triple-pulse operation (left) showing the three pulse profiles (yellow) relative to the Q-switch triggers (red). The profile of the first pulse (left) indicates about $100 \mathrm{nsec}$ pulse-width. 


\subsection{IPDA Receiver}

The 2- $\mu \mathrm{m}$ triple-pulse IPDA lidar receiver is shown in figure 5. The receiver consists of a telescope that focuses the radiation onto $300 \mu \mathrm{m}$ diameter spot. Similar to the double-pulse IPDA, the receiver telescope design is a Newtonian type with $0.4 \mathrm{~m}$ diameter aluminum primary mirror. The shape of the primary mirror is hyperbolic to minimize the aberration and focus the return signal to less than $300 \mu \mathrm{m}$ diameter spot size. The telescope is designed to maintain the focus point position in the temperature range between 5 and $35^{\circ} \mathrm{C}$. The telescope secondary mirror is a two surface dichroic flat that turns the return radiation $90^{\circ}$ to the side integrated aft-optics from one surface. The opposite surface is used to transmit the expanded laser beam coaxially with the telescope. A single automated mount is used for bore-sight alignment. Currently, a second telescope is under integration for this mission, maintaining the integrity of the doublepulse IPDA for further applications.

The telescope focused radiation is collimated, filtered then applied to a beam splitter. The beam splitter generates two optical channels. The first is a high signal channel with $90 \%$ of the radiation applied to a focusing lens on to a $300 \mu \mathrm{m}$ diameter InGaAs pin detector (Hamamatsu; G5853). The detector output is amplified through a variable gain transimpedance amplifier (TIA) (FEMTO; DHPCA- 100). This detection channel is similar to the double-pulse IPDA instrument and it is included as a reference. The second low signal channel, with $10 \%$ radiation, is focused down to accommodate any other detector for IPDA lidar detection investigation. Currently, it is planned to be used with an electron avalanche photodiode (e-APD) detector. Recent development of advance single-charge-carrier, HgCdTe (MCT) e-APD indicated a breakthrough in lidar detection technology. These devices are space-qualifiable and were validated for airborne lidar operation at 1.6- $\mu \mathrm{m}$ at NASA Goddard Space Flight Center (GSFC) [12-13]. In co-ordination with ESTO, LaRC is collaborating with GSFC to integrate their detector into the IPDA low signal channel. This e-APD comes with 4 by 4 pixel format ( $80 \mu \mathrm{m}$ by $80 \mu \mathrm{m}$ pixel area) with read-out electronics that enable access to each pixel through individual TIA. An output summing amplifier would produce the sum of specific number of pixels as selected by the operator. The detector is integrated inside a vacuum chamber that allows cooling the device with cryo-cooler down to $70 \mathrm{~K}$ to reduce dark current and noise. The e-APD and readout electronics are integrated inside a rack mountable chassis. Additional custom designed aft-optics would allow focusing the radiation onto selected number of pixels. The e-APD custom aft-optics is coupled to the IPDA aft-optics through a 2- $\mu \mathrm{m}$ optical fiber, as shown in figure 5. Work efforts at GSFC included 2- $\mu \mathrm{m}$ cold filter integration to the detector chamber, detector assembly testing, additional aft-optics design with optical fiber coupling. Airborne detector chassis assembly and testing are currently progress. Combining both the 2- $\mu \mathrm{m}$ triple-pulse transmitter with this new detection system in a single instrument will result in a $\mathrm{CO}_{2}$ IPDA lidar with enabling technology that meets or exceed space requirement [13].

After amplification, the IPDA detected signals are digitized and stored through a data acquisition unit. The data acquisition unit is based on two similar high-performance digitizers (Agilent; U5303A). These are 12-bit two-channel digitizers that operate at a fixed sampling rate of $1 \mathrm{GS} / \mathrm{s}$. These digitizers are triggered from the laser Q-switch and come with a self-calibration option that is activated for gain and offset measurement and correction for every record. One digitizer is dedicated to the IPDA return signals, with a variable record length of about 70k samples, while the other is dedicated to the laser energy monitors, with a fixed record length of $10 \mathrm{k}$. Using these digitizers is another major enhancement of the triple-pulse IPDA over the double-pulse. Although, digitized data have 12-bit resolution, data storage is achieved in byte increments, leading to 16 -bit data record. This indicates that $25 \%$ of the recorded data would be null. Therefore, optional sample averaging take advantage of some of the extra 4-Bits, while enhancing the data record precision, lowering noise and reducing data volume, as indicated in figure 6. For example, a 1 second worth of data at $50 \mathrm{~Hz}$ repetition rate would results in 150 laser pulses. For each pulse, 4 records are obtained, two from two different energy monitors and another two from the two different detection channels. This would translate to 600 records. Taking into account the record length and two-byte requirement per sample would result in $48 \mathrm{M}$-Byte/sec data rate. Thus, for an average two hours flight the total data collected would be 344 GByte, at $1 \mathrm{GHz}$ sampling rate. The digitizer's data acquisition software was compiled with LabView and output data is stored in MATLAB compatible binary files that includes the raw data and housekeeping. The completed triple-pulse IPDA data acquisition software includes the sample average data reduction algorithms. In addition, work is currently focused on real time data processing that allows the operator to select the recorded data either in raw format, processed format or both. Digitizer's software was tested using an IPDA simulated inputs, as shown in figure 7 along with the data acquisition GUI. Moreover, following IEEE standard, each channel of both digitizers will be characterized to quantify key performance parameters such as noise, cross-talk, harmonic distortion and linearity, that may affect the IPDA performance. Once implemented, the final data acquisition system will be tested with double-pulse IPDA system. 


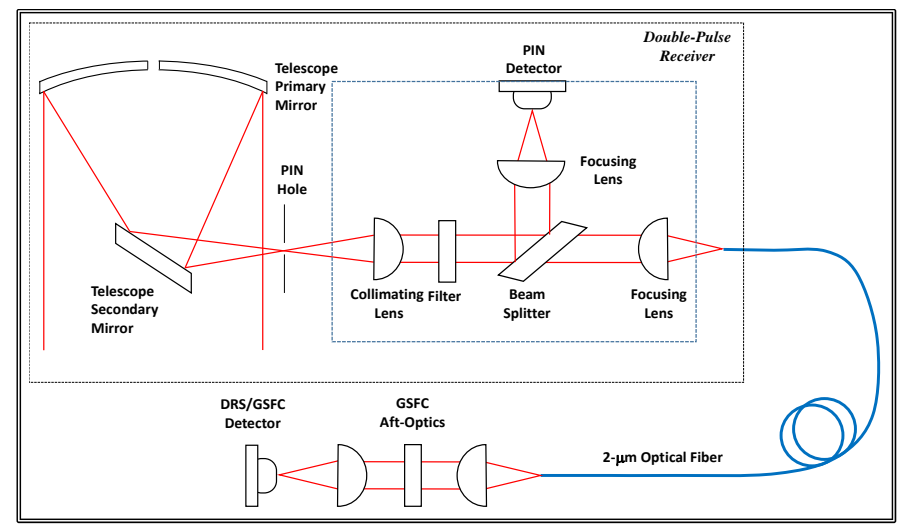

Figure 5. 2- $\mu \mathrm{m}$ triple-pulse IPDA receiver system, integrated based on the previous double-pulse system. The receiver consists of a telescope, aft optics with high-signal InGaAs channel and low-signal e-APD channel. The e-APD channel is coupled through an optical fiber through additional aft-optics.

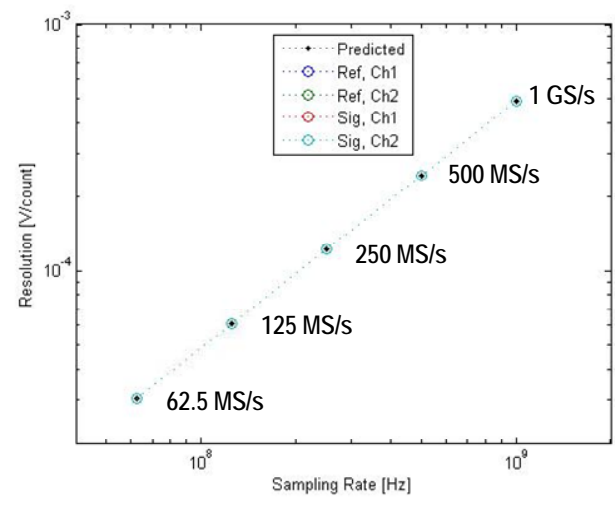

Figure 6. Measurement of the sampling resolution versus sampling rate for all four channels of the two digitizers selected for the triple-pulse IPDA liadr data acquisition. The sampling rate was reduced by sample averaging of the $1 \mathrm{GS} / \mathrm{s}$ acquired data. The predicted resolution is the result of the ratio of the digitizer full scale range of $2 \mathrm{~V}$ to the total number of code obtained from $2^{\mathrm{N}}$, where $\mathrm{N}$ is the number of bits.

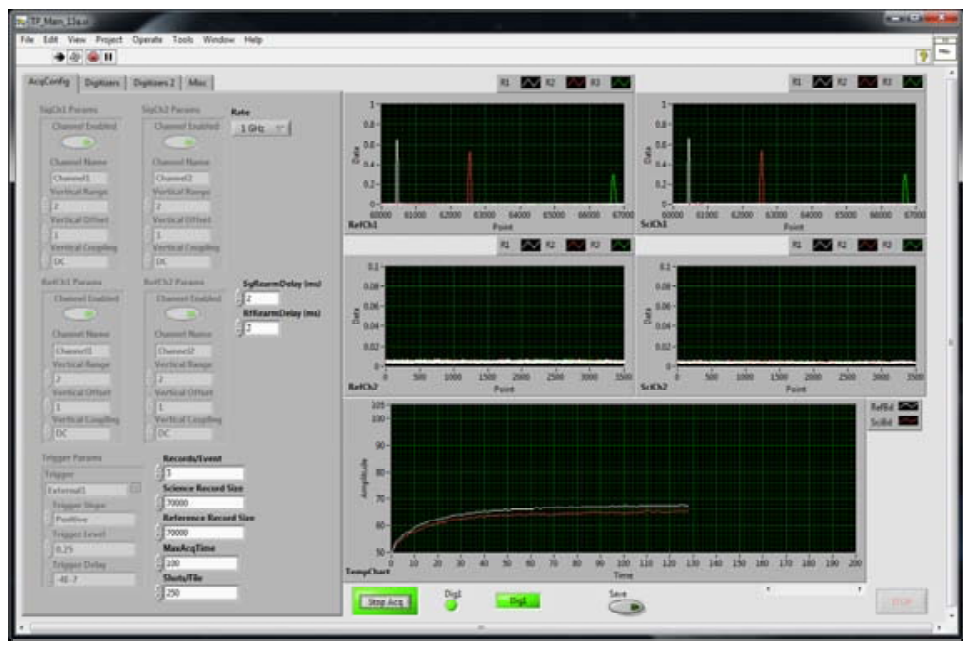

Figure 7. Graphical user interface of the data acquisition software showing the IPDA simulated signals and the digitizers monitored temperature. 


\subsection{IPDA integration}

The 2- $\mu \mathrm{m}$ double-pulse IPDA lidar instrument is installed inside a mobile laboratory, shown in figure 8 . This mobile laboratory is equipped with two access windows. This allows the IPDA instrument to operate either in a horizontal direction through a side-window or zenith through a top window. Although the IPDA itself is pointing nadir, horizontal or zenith pointing is obtained through 24 inch $45^{\circ}$ flat mirrors installed underneath the telescope to turn both the transmitted beam and field-of-view, as shown in the same figure [6]. Aiming horizontal would allow pointing the IPDA to a set of calibrated hard targets for instrument testing and verification, while aiming zenith allows clouds targets. The $2-\mu \mathrm{m}$ double-pulse IPDA lidar system is an ideal test bed for different components of the triple-pulse system. This is achieved by replacing such components while observing the instrument performance. This is applicable to the laser timing control unit for triple-pulse operation, seeding and seeding and wavelength control testing, triple-pulse laser transmitter testing and alignment, receiver detectors (e-APD) verification, energy monitor calibration, digitizers testing and software updates and finally the whole triple-pulse IPDA ground testing and validation. The IPDA mobile laboratory will be set almost in the same double-pulse validation location at LaRC. Figure 9 shows an aerial picture of the test site that includes several advantages. Collocated in the site is the Chemistry and Physics Atmospheric Boundary Layer Experiment (CAPABLE). CAPABLE is a ground-based observation site for studying atmospheric conditions in the Tidewater region of Virginia, which operates through collaborative effort between the Science Directorate at NASA LaRC, the U.S. Environmental Protection Agency and the Virginia Department of Environmental Quality [14]. CAPABLE instruments continuously measure pollutants, such as ozone, nitrogen dioxide, carbon monoxide and aerosols. Beside, CAPABLE provides continuous ground meteorological monitoring, such as pressure, temperature and relative humidity. This data is valuable for the $2-\mu \mathrm{m}$ IPDA lidar modelling for instrument validation on ground. In addition, an in-situ $\mathrm{CO}_{2}$ and $\mathrm{H}^{2} \mathrm{O}$ gas analyzer (LiCor; LI-840A) will be installed at fixed location as shown in figure 9. This allows better estimates of the $\mathrm{CO}_{2}$ mixing ratio in the test location for better evaluation of the 2- $\mu \mathrm{m}$ IPDA lidar instrument. Finally, the collocation of the Hampton-NASA Steam Plant [15] provides a $\mathrm{CO}_{2}$ source that would increase the gas mixing ratio above normal for plume detection validation.

Once the triple-pulse IPDA instrument integration and testing inside the mobile laboratory complete, airborne integration would start by transferring the instrument to the aircraft. The $2-\mu \mathrm{m}$ triple-pulse IPDA lidar is designed for integration into a small research aircraft. The IPDA instrument size, weight and power consumption were restricted to the NASA B200 payload requirements. This allows the system to be easily adopted in any larger airborne research platform, such as the NASA DC-8 aircraft, for future missions. In addition to the IPDA lidar, other housekeeping instruments will be integrated into the B-200 aircraft. These included the LiCor in-situ sensor for $\mathrm{CO}_{2}$ dry mixing ratio measurement, GPS for aircraft position, altitude and angles measurements and video recorder for target identification. Besides, aircraft builtin sensors provided altitude, pressure, temperature and relative humidity sampling at the flight position. Time stamps will be adjusted to the GPS global timing. Figure 10 shows the projected IPDA instrument integrated inside the NASA B-200 aircraft.

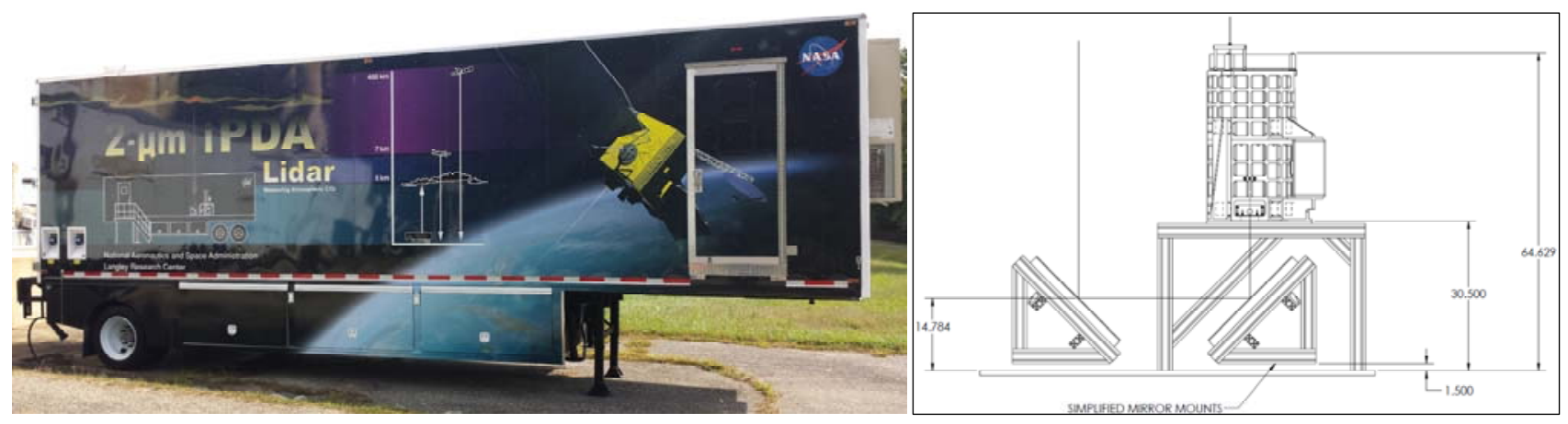

Figure 8. Picture of the IPDA mobile laboratory (left). The mobile laboratory allow instrument horizontal and zenith access using large turn mirrors (right). 


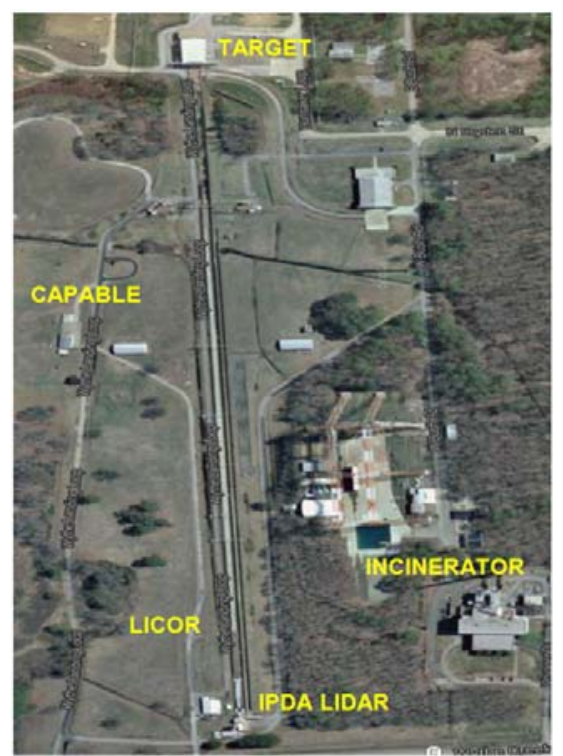

Figure 9. Aerial picture of the 2- $\mu \mathrm{m}$ triple-pulse IPDA ground testing and validation site at NASA LaRC.

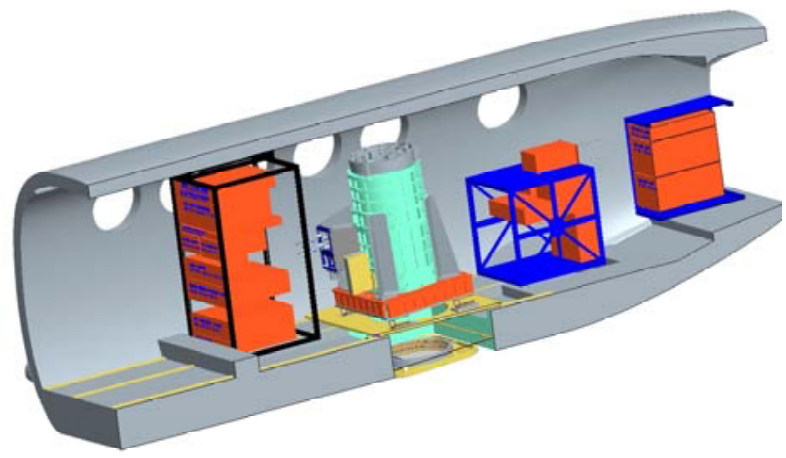

Figure 10. Projection of the 2- $\mu \mathrm{m}$ triple-pulse IPDA instrument integration inside the NASA B-200 aircraft.

\section{VALIDATION PLANS}

The 2- $\mu$ m IPDA lidar validation is important to assess both $\mathrm{CO}_{2}$ and $\mathrm{H}_{2} \mathrm{O}$ atmospheric measurements. IPDA validation is conducted by comparing the instrument measurement to other gas sensors. Instrument validation will start on ground after instrument integration in the mobile laboratory. The mobile laboratory enables IPDA lidar horizontal measurement using a set calibrated hard targets with different reflectivity, in addition to vertical measurements using clouds as hard target. The main ground validation objectives are to check IPDA operational readiness before aircraft deployment, obtain IPDA signals and noise to evaluate instrument systematic and random errors, compare instrument errors to IPDA models and compare $\mathrm{CO}_{2}$ and $\mathrm{H}_{2} \mathrm{O}$ measurements against correlative in-situ instruments, such as CAPABLE, radiosonde, LiCor and Piccaro. Other long term objectives include transferring the laboratory with the instrument to different tall tower sites, such as WLEF tall tower in Park Falls, Wisconsin, and the Southern Great Plains ARM site in Lamont, Oklahoma.

For airborne operation, the objective of the initial engineering flights would focus on instrument operation and comparing the airborne and ground performance, in terms of signal and noise. Once proven success, the objective would focus on comparing $\mathrm{CO}_{2}$ and $\mathrm{H}_{2} \mathrm{O}$ measurements against correlative in-situ sensors and models. The validation will rely on onboard sensors (aircraft sensors, LiCor and Piccaro) as well as coordinating and collaborate with science community from other independent sensors, such as NOAA air-sampling flask flights and Pennsylvania State University passive sensors. The main goal is to evaluate and verify both the systematic and the random errors. On the other hand, airborne 
validation will be planned to target different conditions such as different surface reflectivity (land, ocean, snow, sand, vegetation) uniform versus variable surface reflectivity, day and night background, clear, cloudy and broken cloud conditions, variable surface elevation and urban pollution and plume detection. The validation may also cover different location such as the Upper Midwest summer, where strong vertical and horizontal spatial gradients in $\mathrm{CO}_{2}$ occurs due to agricultural fluxes and urban deployment in winter, where flight around isolated urban centers could identify a clear atmospheric boundary layer $\mathrm{CO}_{2}$ plume (for example, Indianapolis, build on existing in situ observational network). Also, these $2-\mu \mathrm{m}$ triple-pulse validation activities might have the potential to coordinate with ACT-America flights.

\section{SUMMARY AND CONCLUSIONS}

A 2- $\mu \mathrm{m}$ triple-pulse IPDA lidar instrument is being developed at NASA LaRC. This active remote sensing IPDA instrument targets and measures both atmospheric carbon dioxide and water vapor. Wavelength selection and laser transmitter operation allows measuring both species independently and simultaneously. This is the first demonstration of measuring two different atmospheric molecules with a single instrument. The basic instrument design is based on knowledge gathered through the previously successful $2-\mu \mathrm{m}$ double-pulse IPDA. Critical enhancements were implemented in the new triple-pulse design that significantly advance the technology. These enhancements includes both the transmitter and receiver. For the transmitter, modifications include triple-pulse operation of the laser, laser timing control updates and wavelength control design. In the receiver, updates includes the data acquisition system and additional high performance e-APD detector. The e-APD detector supplied by NASA GSFC, is a state-of-art, space qualifiable device that was validated for lidar applications. Combining both the $2-\mu \mathrm{m}$ triple-pulse transmitter with this new detector in a single instrument will result in a $\mathrm{CO}_{2}$ IPDA lidar with enabling technology that meets or exceed space requirement. Work progress of the $2-\mu \mathrm{m}$ triple-pulse IPDA program is on schedule. Instrument validation plans are under discussions to collaborate with different institutes with similar interests.

\section{ACKNOWLEDGMENTS}

This work was funded and supported by NASA Earth Science Technology Office (Program Director: George Komar and Program Manager: Parminder Ghuman).

\section{REFERENCES}

[1] U. Singh, B. Walsh, J. Yu, M. Petros, M. Kavaya, T. Refaat, and N. Barnes, "Twenty years of Tm:Ho:YLF and $\mathrm{LuLiF}$ laser development for global wind and carbon dioxide active remote sensing", Optical Materials Express, 5, $827,2015$.

[2] G. Koch, J. Beyon, F. Gibert, B. Barnes, S. Ismail, M. Petros, P. Petzar, J. Yu, E. Modlin, K. Davis and U. Singh, "Side-line tunable laser transmitter for differential absorption lidar measurements of $\mathrm{CO}_{2}$ : design and application to atmospheric measurements", Applied Optics 47, 944-956, 2008.

[3] T. Refaat, S. Ismail, G. Koch, M. Rubio, T. Mack, A. Notari, J. Collins, J. Lewis, R. De Young, Y. Choi, N. Abedin, and U. Singh, "Backscatter 2- $\mu \mathrm{m}$ lidar validation for atmospheric $\mathrm{CO}_{2}$ differential absorption lidar applications," IEEE Trans. Geosci. Remote Sens. 49, 572-580, 2011.

[4] T. Refaat, S. Ismail, G. Koch, L. Diaz, K. Davis, M. Rubio, M. Abedin, and U. Singh, "Field testing of a twomicron DIAL system for profiling atmospheric carbon dioxide," in Proceedings of the 25th International Laser Radar Conference, St. Petersburg, Russia, 2010.

[5] J. Yu, A. Braud, and M. Petros, “600-mJ, double-pulse 2- $\mu \mathrm{m}$ laser," Optics Letters 28, 540-542, 2003.

[6] U. Singh, T. Refaat, J. Yu, M. Petros and R. Remus, "Airborne active remote sensor for atmospheric carbon dioxide", SPIE Newsroom, 2015.

[7] T. Refaat, U. Singh, M. Petros, R. Remus and J. Yu, "Self-calibration and laser energy monitor validation for a double-pulsed 2- $\mu \mathrm{m} \mathrm{CO}_{2}$ integrated path differential absorption lidar application", Applied Optics 54, 7240-7251, 2015.

[8] U. Singh, J. Yu, M. Petros, T. Refaat, R. Remus, J. Fay and K. Reithmaier, "Airborne 2-micron double-pulsed integrated path differential absorption lidar for column $\mathrm{CO}_{2}$ measurement", Proc. of SPIE 9246, 924602, 2014.

[9] U. Singh, T. Refaat, J. Yu, M. Petros and R. Remus, "Double-pulsed 2- $\mu \mathrm{m}$ lidar validation for atmospheric $\mathrm{CO}_{2}$ measurements", Proc. of SPIE 9645, 964502, 2015. 
[10]U. Singh, J. Yu, M. Petros, T. Refaat, R. Remus and K. Reithmaier, "Development of double- and triple-pulsed 2micron IPDA lidars for column $\mathrm{CO}_{2}$ measurements", Proc. of SPIE 9612, 961204, 2015.

[11] T. Refaat, U. Singh, J. Yu, M. Petros, S. Ismail, M. Kavaya, and K. Davis, "Evaluation of an airborne triple-pulsed 2- $\mu \mathrm{m}$ IPDA lidar for simultaneous and independent atmospheric water vapor and carbon dioxide measurements", Applied Optics 54, 1387-1398, 2015.

[12] X. Sun, J. Abshire, J. Beck, 2014: HgCdTe e-APD detector arrays with single photon sensitivity for space lidar applications, Proc. of SPIE 9114, 91140K, 2014.

[13] U. Singh, T. Refaat, M. Petros and J. Yu, "triple-pulsed two-micron integrated path differential absorption lidar: a new active remote sensing capability with path to space," in Proceedings of the 27th International Laser Radar Conference, New York, NY, 2015.

[14] capable.larc.nasa.gov

[15] www.hampton.gov 